\title{
Co-Existence of ITS-G5 and C-V2X at an Urban Road Intersection
}

\author{
Sandaruwan Jayaweera, Shashika Manosha, Nandana Rajatheva and Matti Latva-aho \\ Centre for Wireless Communications, Univeristy of Oulu, Finland \\ E-mail: \{sandaruwan.jayaweera, nandana.rajatheva, matti.latva-aho\}@oulu.fi \\ manoshadt@gmail.com
}

\begin{abstract}
High mobility, low latency and high throughput requirements in intelligent transport systems (ITS) have paved the way for the development of new wireless communication technologies. Therefore, the $5.9 \mathrm{GHz}$ band has been assigned to ITS applications under two main technologies. Europe's ITS-G5 is one such technology, which is based on IEEE 802.11p. The other alternative technology is 3GPP's cellular vehicleto-everything (C-V2X). Both of these technologies have their inherent advantages and disadvantages due to dissimilarities in their physical (PHY) and media access control (MAC) layer architectures. Therefore, the applicability of each technology will vary depending on the situation. While previous work has been mainly focused on the comparison of two technologies, in this paper, we investigate the benefit of the co-existence of both of these technologies for a V2I downlink scenario in a road-side unit (RSU) placed at the center of an urban road intersection. We propose an optimization scheme to achieve the best minimum signal-tointerference-plus-noise ratio (SINR) performance for the RSU while providing connectivity to the maximum possible number of vehicles. Our analysis shows that ITS-G5 should be given priority when communicating with larger number of vehicles while C-V2X should be given priority when less number of vehicles are requesting to connect with the RSU.

Index Terms - Intelligent transport systems, ITS-G5, IEEE $802.11 \mathrm{p}$, 3GPP, cellular vehicle-to-everything, physical layer, media access control layer, downlink, road-side unit.
\end{abstract}

\section{INTRODUCTION}

In modern ITS solutions, on-board units (OBU) placed in vehicles communicate with nearby vehicles equipped with OBUs to provide direct vehicle-to-vehicle (V2V) communication. In addition to the $\mathrm{V} 2 \mathrm{~V}$ communication, ITS solutions contain vehicle-to-infrastructure (V2I) communication which is supported by the deployment of road-side units (RSU) in critical places within city infrastructure. Two main technology standards have emerged in recent years to support these communication scenarios. The dedicated short-range communication (DSRC) protocol developed in US [1] and the ITS-G5 protocol developed in Europe [2] are similar technologies which have been established based on IEEE 802.11p standard. The other technology, cellular V2X (C-V2X) was introduced in third generation partnership project's (3GPP) Release 14 and was evolved in Release 15 [3] and Release 16.

There have been many research investigations carried out to optimize or to reduce the load of both ITS-G5 and C-V2X independently. Sadio et al. in [4] suggest using Wifi
Direct as a second communication alternative to transfer non-critical data. Furthermore, several studies considered the comparison and co-existence of ITS-G5 and C-V2X. In [5], a comparison of the PHY layers and associated MAC layers of these technologies has been carried out to determine the performance for different vehicle densities. In [6], it discusses a cooperative awareness message (CAM) forwarding mechanism which takes advantage of dual interfaced vehicles which can forward packets between DSRC and C-V2X. Wang et al. in [7] study a spectrum sharing problem where cellular V2X users coexist with Vehicular ad-hoc network (VANET) users in the unlicensed spectrum.

According to the ETSI harmonized standard EN 302 571 [8], the ITS spectrum is segmented into 3 parts:

- 5855-5875 MHz for non-safety ITS applications;

- 5875-5905 MHz for safety-related ITS applications;

- 5905-5925 MHz for Future ITS applications.

The 5G Automotive Association suggests a priory agreement to split the $5.9 \mathrm{GHz}$ safety related ITS spectrum between two technologies where separate $10 \mathrm{MHz}$ channels at each end of the spectrum are reserved for each technology while keeping the channel in the middle vacant [9]. It is suggested to develop mutual detect-and-vacate algorithms for the two technologies to access the middle part of the spectrum. Although this opens up the window for a possible co-existence in the future, without proper consideration about each technology's strengths and weaknesses, there is a possibility for the ITS spectrum to be under-utilized.

With the rapid developments in the vehicular industry, there is a possibility to see autonomous driving cars in the near future [10]. These vehicles will require efficient and reliable communication networks which will be responsible for communicating huge amounts of data collected from a variety of sensors such as LiDAR, radar, and high-quality cameras [11]. It is very important for vehicular networks to have the ability to work without interruptions with such large amounts of data since vehicular communication has the potential to reduce or control vehicle-related accidents and minimize the fuel and other resource wastage. In urban regions, due to high traffic, there is a possibility for vehicular communication networks to be overloaded with data. In particular, road intersections will become the center for vehicle clusters [12]. Cho et al. in [13] suggest a vehicle distribution in an urban area where vehicles to be 
TABLE I

Performance of selected modulation and coding schemes $(10 \mathrm{~ms} / 10 \mathrm{MHz}$ channel).

\begin{tabular}{|c|c|c|c|c|c|c|}
\hline \multirow[t]{2}{*}{ Class } & \multicolumn{3}{|c|}{ ITS-G5 } & \multicolumn{3}{|c|}{$\mathrm{C}-\mathrm{V} 2 \mathrm{X}$} \\
\hline & $\begin{array}{c}\text { Minimum SINR } \\
(\mathrm{dB})\end{array}$ & Transmitted packets & Range (m) & $\begin{array}{l}\text { Minimum SINR } \\
(\mathrm{dB})\end{array}$ & Transmitted packets & Range (m) \\
\hline 1 & 27 & 73 & 84 & 28.1 & 50 & 137 \\
\hline 2 & 26 & 69 & 89 & 25.9 & 50 & 150 \\
\hline 3 & 22 & 56 & 112 & 21.1 & 50 & 191 \\
\hline 4 & 18 & 40 & 141 & 18.7 & 40 & 213 \\
\hline 5 & 15 & 32 & 167 & 14.5 & 30 & 258 \\
\hline 6 & 13 & 22 & 188 & 12.8 & 30 & 278 \\
\hline 7 & 11 & 17 & 210 & 11.2 & 20 & 294 \\
\hline 8 & 10 & 11 & 223 & 9.9 & 20 & 306 \\
\hline
\end{tabular}

distributed in clusters.

Therefore, the efficient management of V2X communication traffic at urban intersections will become a definite concern in the vehicular communication system development and urban planning. The developers will be further constrained by the limited spectrum resources allocated to ITS applications. Since both ITS-G5 and C-V2X offer their own set of benefits and drawbacks, a solution can be obtained by the proper utilization of these technologies.

In addition, the V2I downlink communication is often affected by the leakage interference from V2V communication occurring in adjacent channels [14]. The effect of this leakage interference depends on the communication technology used in the V2I communication. Therefore, a proper channel allocation scheme should be introduced to minimize the effect of this adjacent channel interference $(\mathrm{ACI})$

In this paper, We use the term base station (BS) and RSU to refer the same infrastructure within the ITS network. We study the downlink V2I communication at a single road intersection with an RSU placed at the center. We assume the roads are perpendicular to each other in urban environments. We model the RSU to communicate with a set of vehicles selected from the population. We describe a scheme that maximize the SINR performance of the vehicle with the weakest channel while using both C-V2X and ITS-G5 to achieve connectivity. We present a method to figure out the technology most suitable for each vehicle. The technology selection scheme is modeled and solved by using a MATLAB/CVX package. Additionally, we suggest a channel allocation scheme to minimize the effect of leakage interference from the $\mathrm{V} 2 \mathrm{~V}$ communication towards the intended V2I communication.

The rest of this paper can be summarized as follows. Section II elaborates on the PHY layer of the two technologies, the system model and the problem formulation. The algorithm for optimum co-existence and the channel allocation scheme for ACI minimization is described in Section III. We analyze the optimum co-existence scenarios for the two technologies and ACI minimization using numerical simulation results in Section IV. Finally, Section $\mathrm{V}$ concludes the paper.

\section{System Model and Problem Formulation}

\section{A. Technology Overview}

The PHY layer of ITS-G5 adopts orthogonal frequency division multiplexing (OFDM) with a symbol duration of $8 \mu \mathrm{s}$ and a subcarrier spacing of $156.25 \mathrm{kHz}$. The channel bandwidth requirement is $10 \mathrm{MHz}$. ITS-G5 supports eight modulation and coding schemes (MCSs). In comparison, the PHY layer of C-V2X uses single carrier frequency division multiple access (SC-FDMA). Total duration for 14 symbols is $1 \mathrm{~ms}$, which is called a transmission time interval (TTI) and the subcarrier spacing is $15 \mathrm{kHz}$. Twelve subcarriers will form a resource block (RB) which is the smallest resource that can be allocated to a user. A $10 \mathrm{MHz}$ channel has the ability to support 50 RBs. Unlike an ITS-G5 channel which can support only one transmission in a single instance, $\mathrm{C}-\mathrm{V} 2 \mathrm{X}$ has the ability to support multiple parallel transmissions in a single instant by properly handling the RBs. Therefore, C-V2X has a better flexibility in the resource allocation in frequency domain. But due to high symbol duration, C-V2X transmission rate is less than ITS-G5 at high MCS indexes. For different modulation and coding schemes (MSCs) and a message size of 300 bytes, the SINR, range and latency performances of these technologies are calculated in [15].

Using the performance values obtained from [15], we can calculate the number of messages transmitted and the transmission range for a $10 \mathrm{~ms}$ time period and $10 \mathrm{MHz}$ channel bandwidth. For approximately similar minimum SINR performances, we can categorize the different MSCs of two technologies into 8 different classes. These details are listed in Table I.

\section{B. Vehicle Distribution Model and Optimization Problem}

Let the indicator $\theta_{i}$ define technology selection for the $i$ th vehicle which is defined as,

$$
\theta_{i}= \begin{cases}1 & \text { if ITS-G5 } \\ 0 & \text { if C-V2X. }\end{cases}
$$

We consider a downlink V2I communication scenario with the transmitter located at the center and the $i$ th 
TABLE II

ACIR values for different V2X technologies [14].

\begin{tabular}{|c|c|c|c|c|}
\hline Aggressor & Victim & ACLR [dBc] & ACS [dB] & ACIR [dB] \\
\hline ITS-G5 & ITS-G5 & 26 & $22-29$ & $20.6-24.5$ \\
C-V2X & ITS-G5 & 30 & $22-29$ & $21.4-26.5$ \\
ITS-G5 & C-V2X & 26 & 33 & 25.2 \\
C-V2X & C-V2X & 30 & 33 & 28.2 \\
\hline
\end{tabular}

vehicle or receiver situated $r_{i}$ distance away from the transmitter. The received power with LOS path-loss is,

$$
P_{i}=\theta_{i} r_{i}^{-\alpha_{I T S}} P^{I T S}+\left(1-\theta_{i}\right) r_{i}^{-\alpha_{C V 2 X}} P^{C V 2 X},
$$

where the notations $\alpha_{I T S}$ and $\alpha_{C V 2 X}$ represent the pathloss exponents of ITS-G5 and C-V2X, respectively. The transmit power at the BS for ITS-G5 is $P^{I T S}$ and $P^{C V 2 X}$ is the transmit powers at the $\mathrm{BS}$ for $\mathrm{C}-\mathrm{V} 2 \mathrm{X}$.

We introduce $\theta=\left[\theta_{1}, \theta_{2}, \ldots, \theta_{i}, \ldots, \theta_{N}\right]$ for the technology selection of all $\mathrm{N}$ vehicles connecting with the BS. Since the BS has the ability to allocate resources among the users, we assume that there is no V2V communication or V2I communication sharing the same channel. Therefore there will be no interference. Therefore, the SINR at $i$ th vehicle can be defined as,

$$
\gamma_{i}=\frac{\theta_{i} r_{i}^{-\alpha_{I T S}} P^{I T S}+\left(1-\theta_{i}\right) r_{i}^{-\alpha_{C V 2 X}} P^{C V 2 X}}{\sigma^{2}},
$$

where the minimum SINR maximization is achieved by choosing the optimum $\theta$ value for all $\mathrm{i}=1,2 \ldots, \mathrm{N}$. Moreover, the usable bandwidth for the BS is limited by $B_{t o t}$. This optimization problem can be formulated as,

$$
\begin{array}{ll}
\max _{\Theta} \min _{i \in[1, N]} \gamma_{i} \\
\text { subject to } & \left\lceil\frac{\sum_{i=1}^{N} \theta_{i}}{N_{I T S}}\right\rceil+\left\lceil\frac{\sum_{i=1}^{N}\left(1-\theta_{i}\right)}{N_{C V 2 X}}\right\rceil \leqslant B_{t o t} \\
& \theta_{i} \in\{0,1\}, \text { for } \mathrm{i}=1,2, \ldots, \mathrm{N},
\end{array}
$$

where $\theta_{i}$ for all $\mathrm{i}=1,2, \ldots, \mathrm{N}$ are the optimization variables. The number of packets transmitted in $10 \mathrm{~ms}$ by ITSG5 channel and C-V2X channel are $N_{I T S}$ and $N_{C V 2 X}$, respectively. C-V2X has the advantage of having a larger range. This can be observed in Table I. Therefore, C-V2X has the higher SINR performance at the receiver. In high rate MCS scenarios, $N_{I T S}>N_{C V 2 X}$. Therefore, ITS-G5 is able to provide connectivity to more vehicles than C-V2X.

Therefore, when the resource availability is high and demand is low, $\mathrm{C}-\mathrm{V} 2 \mathrm{X}$ is given higher priority. But as the demand increases or resources decrease, allocation to ITSG5 begins to increase. This prevents the trivial solution of only one technology being preferred over the other in every instance and generates a proper co-existence scenario.

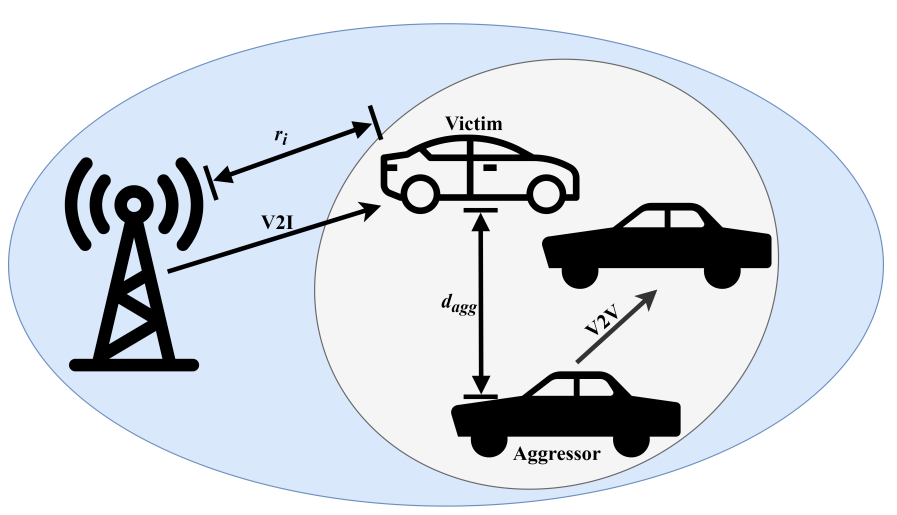

Fig. 1. Scenario where V2V communication causing ACI on V2I communication.

\section{Leakage Interference Model}

There is a probability of a leakage interference from the $\mathrm{V} 2 \mathrm{~V}$ communication in neighbouring channels. The main parameter to consider in leakage interference measurement is the adjacent channel interference ratio (ACIR), which is defined as the ratio of the total power from the assigned channel to the total interference power affecting a victim vehicle. The ACIR can be obtained using,

$$
A C I R \approx \Phi=\frac{1}{\frac{1}{A C L R}+\frac{1}{A C S}},
$$

where, adjacent channel leakage ratio $(A C L R)$ is defined as the ratio of the transmitted power to the power measured in the adjacent channel. Adjacent channel selectivity $(A C S)$ is defined as the ratio of the receiver filter attenuation on the assigned channel to that on the adjacent channel. Different ACLR, ACS and ACIR values for different V2X technologies are given in Table II. The V2V communication acts as an aggressor towards V2I communication. Therefore, V2V communication technology is considered as the aggressor while V2I communication technology is considered as the victim. Similar to the previous situation, the distance from the BS to the victim vehicle is $r_{i}$. The distance from the aggressor to the victim is $d_{a g g}$. This scenario is illustrated in Fig. 1. The leakage interference can be obtained from,

$$
P_{\text {leak }}=P^{a g g} d_{a g g}^{-\alpha_{V 2 V}} 10^{\left(\frac{-\Phi}{10}\right)},
$$

where, $P^{a g g}$ is the transmit power of the aggressor. Therefore, the SINR for the $i^{\text {th }}$ vehicle can be obtained from,

$$
\gamma_{i}=\frac{\theta_{i} r_{i}^{-\alpha_{I T S}} P^{I T S}+\left(1-\theta_{i}\right) r_{i}^{-\alpha_{C V 2 X}} P^{C V 2 X}}{\sigma^{2}+P^{a g g} d_{a g g}^{-\alpha_{V 2 V}} 10^{\left(\frac{-\Phi}{10}\right)}} .
$$

\section{Proposed Algorithm And Channel Allocation Scheme}

With high vehicle numbers, there can be certain instances where the optimization problem in (4) becomes 
unsolvable. For example, when the bandwidth constraint is very low, even by using ITS-G5 technology, the BS is unable to allocate resources for all $\mathrm{N}$ vehicles. Therefore, a modification to the initial $N\left(N^{(0)}\right)$ is suggested in the algorithm introduced in this Section. In addition, an example channel allocation scheme to minimize the effect of ACI is suggested.

\section{A. Algorithm Development}

Even when the RSU only allocates resources to the technology which supports higher number of vehicles, it might be insufficient in some situations. In other words, within a single $10 \mathrm{~ms}$ instance, when the number of vehicles expecting to be connected with the BS is higher than both $B_{t o t} \times N_{I T S}$ and $B_{t o t} \times N_{C V 2 X}$, the 1st constraint in the optimization problem becomes unsatisfied. The RSU can identify this situation by the boolean expression is given in,

$$
f_{1}=\left(N>B_{t o t} \times N_{I T S}\right) \&\left(N>B_{t o t} \times N_{C V 2 X}\right) .
$$

Although, $N_{I T S}>N_{C V 2 X}$ in our test case, there is a possibility for $N_{I T S}<N_{C V 2 X}$ according to Table I. Therefore, rather than only using $B_{t o t} \times N_{I T S}$, the compatibility of both technologies is considered. Therefore, only if both technologies are unable to provide sufficient resources for the demand, the value of $N$ is modified.

Since the compatibility of both technologies is considered in (8), the technology which is able to provide maximum connectivity should be identified. Therefore, the values $N_{I T S}$ and $N_{C V 2 X}$ are compared. The technology which provides the maximum connectivity comes up with a higher value. Therefore, the value of $N$ is finalized as,

$$
N= \begin{cases}B_{t o t} \times N_{I T S} & N_{I T S} \geqslant N_{C V 2 X} \\ B_{t o t} \times N_{C V 2 X} & N_{I T S}<N_{C V 2 X} .\end{cases}
$$

Although this appears simple, there should be many factors to be considered before selecting the modified $N$ number of vehicles from the initial client vehicle population $\left(N=N^{(0)}\right)$. For the simplicity, in this research, the vehicles with a stronger link are selected to communicate with the BS. With MAC layer, a scoring scheme could be introduced to increase priority to the vehicles which are rejected. Therefore, later when these rejected vehicles try to establish connections with the BS, they will be given higher priority than other vehicles. Therefore, it will be fairer to the vehicles with weak channels. The finalized procedure is illustrated in Algorithm 1.

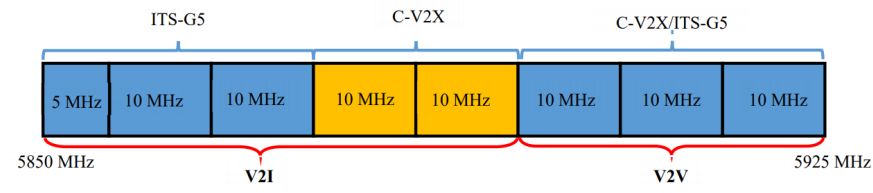

Fig. 2. Resource allocation for technologies while minimizing leakage interference.

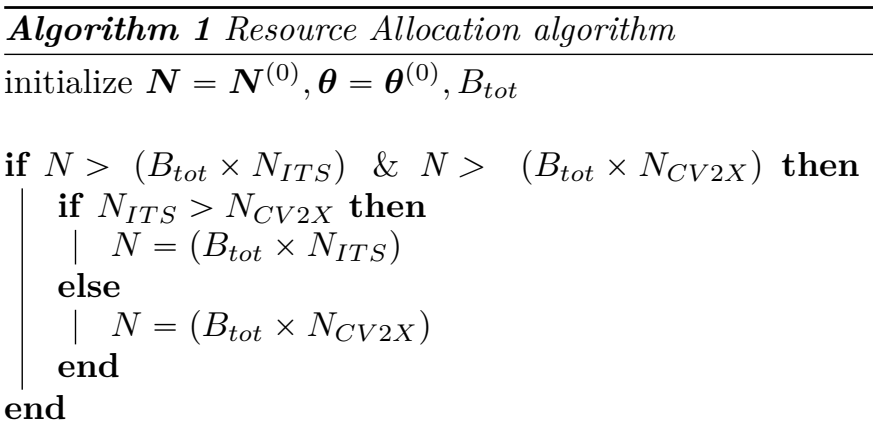

Solve optimization problem in (4) and get $\boldsymbol{\theta}$

\section{B. Channel Allocation Scheme}

According to the the values obtained from Table II, the ACIR is higher when the victim technology is C-V2X. Therefore, when there is ACI caused by V2V communication, the adjacent channels with V2I links should be allocated with C-V2X technology. From the RSU's point of view, when a portion of the spectrum is allocated for $\mathrm{V} 2 \mathrm{~V}$ communication, the BS should allocate C-V2X technology to the channels adjacent to V2V channels. Such an allocation is shown in Fig. 2.

\section{Numerical Results}

In this section, we present the numerical results of our optimization problem in (4) and SINR comparison for different victim technologies under ACI as modeled in (7). We present the number of resources allocated to the ITSG5 and C-V2X technologies under fixed bandwidth and client population $(N)$ scenarios. Additionally, we plot the SINR under ACI against the distance to a single aggressor for both V2I communication technologies.

\section{A. Co-existence Optimization Analysis}

The vehicles distances are generated under a Poisson distribution. The maximum usable bandwidth for ITS in the $5.9 \mathrm{GHz}$ spectrum is $75 \mathrm{MHz}(5850-5925 \mathrm{MHz})$. Therefore, the allocated spectrum has the ability to support seven $10 \mathrm{MHz}$ channels. The transmit power $P_{i}^{I T S}$ $=P_{i}^{C V 2 X}=23 \mathrm{dBm}$. According to the values listed in Table I, the SINR of ITS-G5 at these distances below $300 \mathrm{~m}$ is obviously lower than C-V2X. In our work, the path-loss exponents $\alpha_{I T S}=4$ and $\alpha_{C V 2 X}=3$. The values are obtained by fitting the SINR and distance values from Table I into power models. The value of $\alpha_{I T S}$ can be further verified by [16]. 


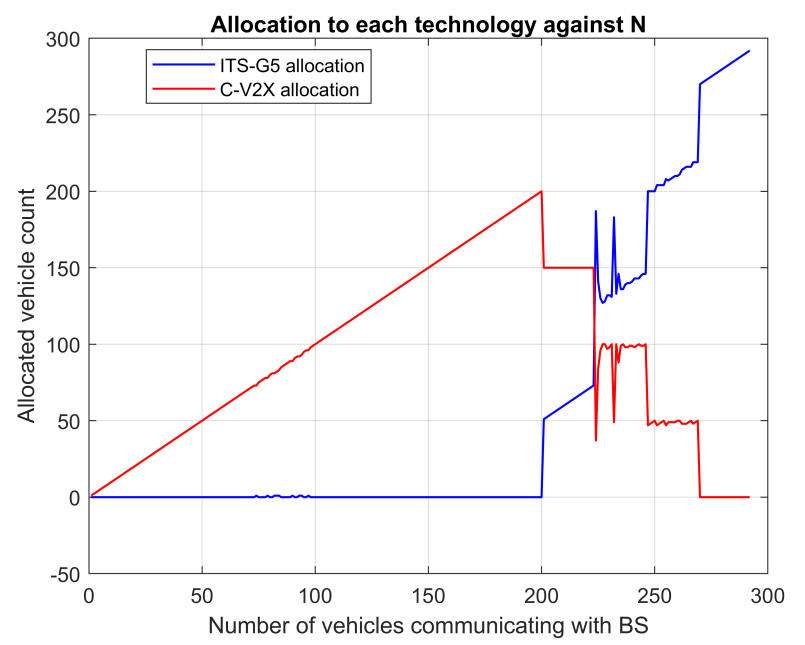

Fig. 3. Allocated number of vehicles for ITS-G5 and C-V2X against the number of vehicles communicating with the BS $(N)$.

While solving the optimization problem, we need to provide maximum connectivity to the vehicles in the population. We need the MCS classes with highest rates to achieve this. Therefore, class 1 from Table I is used. The number of packets transmitted during $10 \mathrm{~ms} N_{I T S}=73$ and $N_{C V 2 X}=50$. The transmitted packet numbers $N_{I T S}$ and $N_{C V 2 X}$ are allocated to satisfy the $10 \mathrm{MHz}$ channel specifications.

The allocation of resources for ITS-G5 and C-V2X for a various number of client vehicles is shown in Fig. 3. The number of connections is varied from 0 to 292 . the simulation has a fixed bandwidth constraint $B_{t o t}=4$. As it can be observed, the number of allocations for $\mathrm{C}-\mathrm{V} 2 \mathrm{X}$ seems to have a gradual increase against the number of vehicles communicating with the BS $(N)$ while the number of allocations for ITS-G5 remains zero. This is due to the fact that the pathloss exponent of $\mathrm{C}-\mathrm{V} 2 \mathrm{X}$ being smaller compared to the pathloss exponent of ITS-G5. Therefore, $\mathrm{C}-\mathrm{V} 2 \mathrm{X}$ is able to deliver much higher SINR values than ITS-G5 at the client receivers. However, as $N$ further increases, allocations for ITS-G5 starts to appear. This is a result of $\mathrm{C}-\mathrm{V} 2 \mathrm{X}$ having a higher bandwidth requirement than ITS-G5. Therefore, with further C-V2X only allocations, there is a possibility of exceeding the bandwidth constraint. This results in an increase of allocations for less bandwidth requiring ITS-G5. Ultimately, with 292 connections, it can be observed that $100 \%$ of vehicles have been allocated with ITS-G5.

Fig. 4 depicts the allocated number of vehicles for each technology against the Bandwidth constraint $\left(B_{t o t}\right)$. Here, the simulation is performed for a fixed client vehicle population, $N=350$. As expected, at low bandwidth situations, the allocations are dominated by low bandwidth requiring ITS-G5. The resources are allocated to ITS-G5 until every client is provided with a channel. A bandwidth of $50 \mathrm{MHz}$ is sufficient to provide all 350 clients with a communication

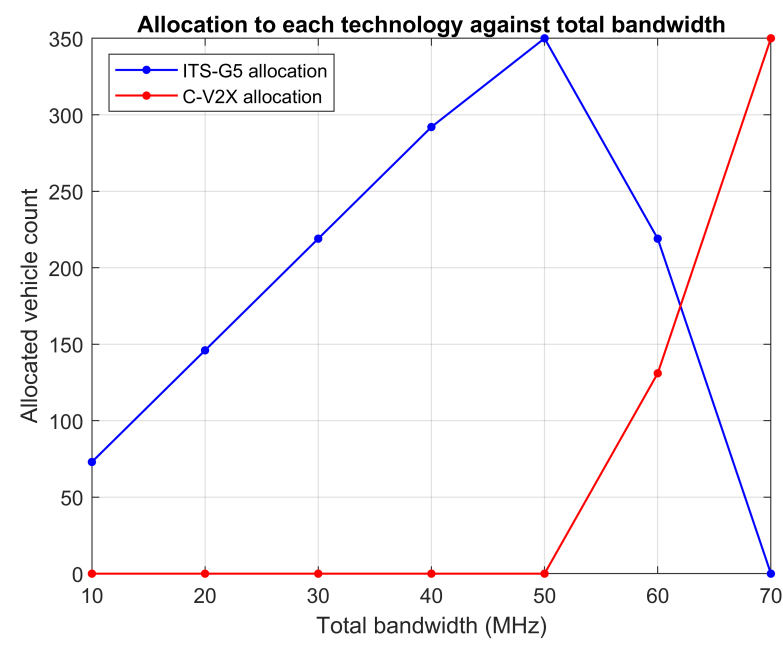

Fig. 4. Allocated number of vehicles for ITS-G5 and C-V2X against the bandwidth constraint.

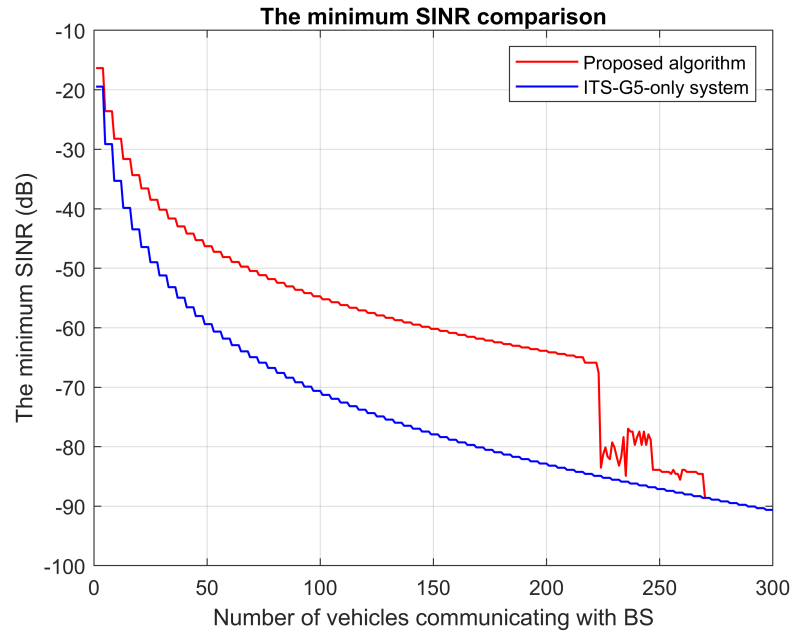

Fig. 5. The minimum SINR against N.

channel with the BS using ITS-G5. With further increases in the bandwidth constraint, ITS-G5 channels begin to be replaced with C-V2X channels. Since C-V2X has better performance at the receiver, this process continues until all 350 clients are allocated with C-V2X channels.

To observe the effect of the algorithm, minimum SINR is plotted against the number of vehicles communicating with the BS. We compare the performance of our system with an all ITS-G5 system. The resulting comparison is addressed through Fig. 5. Obviously, if only C-V2X is used in this situation, the best SINR values can be observed. But, due to resource limitations, C-V2X technology cannot handle high vehicle numbers. Therefore, there is no point in comparing with the minimum SINR of an all C-V2X system. The number of vehicles communicating with the BS varies from 1 to 300 while the bandwidth constraint $B_{t o t}=4$. 


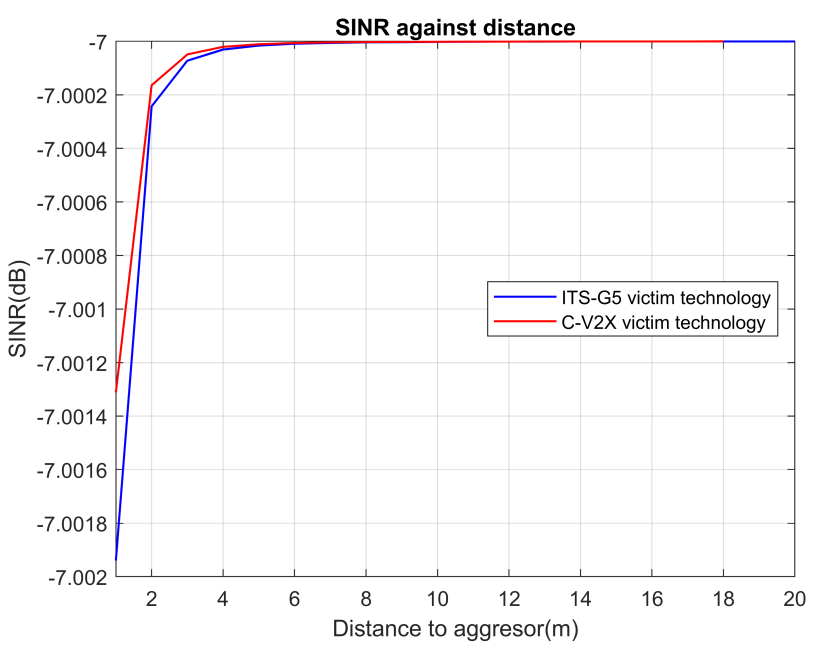

Fig. 6. Comparison of SINR performance of ITS-G5 and C-V2X V2I communication in the presence of ACI.

\section{B. ACI Analysis}

Fig. 6 shows a comparison of the SINR performance of ITS-G5 and C-V2X for a victim vehicle situated $1 \mathrm{~m}$ distance away from the BS. The BS tries to communicate with the victim vehicle using both technologies in two separate instances. The aggressor communication technology is $\mathrm{C}-\mathrm{V} 2 \mathrm{X}$. In our simulation, we change the distance to the aggressor vehicle $\left(d_{\text {agg }}\right)$ from $1 \mathrm{~m}$ to $20 \mathrm{~m}$. As it can be observed, the SINR of the victim increases as the distance to the aggressor increase. But, in each situation, better SINR performance is provided when the victim vehicle uses $\mathrm{C}-\mathrm{V} 2 \mathrm{X}$ as communication technology. A similar result can be observed when ITS-G5 is used as the aggressor technology.

\section{Conclusion}

In this paper, we have analyzed a possible co-existence scenario of two vehicular communication technologies. We have first derived an optimization problem which allowed us to find the number of allocations for each technology while maximizing the SINR of the vehicles with the weakest channels. The optimum co-existence scenarios were evaluated through numerical simulations by plotting the number of allocations for both technologies on the same graphs. As numerical results suggest, with the availability of sufficient resources, C-V2X is able to provide the clients with better SINR values. Therefore, more resources are allocated to $\mathrm{C}-\mathrm{V} 2 \mathrm{X}$ when the demand is less. However, this better performance comes at the expense of high bandwidth demand. Therefore, in high demand situations, the allocation seems to move more towards ITS-G5. With the suggested optimization scheme, the RSU is able to provide higher SINR to the vehicles while providing maximum connectivity. This optimization scheme can be further extended to consider the effect of the maximum delay of each technology. Finally, in the presence of leakage interference from $\mathrm{V} 2 \mathrm{~V}$ communication, $\mathrm{C}-\mathrm{V} 2 \mathrm{X}$ performs better as the downlink V2I technology. Therefore, C-V2X should be allocated to the channels adjacent to $\mathrm{V} 2 \mathrm{~V}$ communication channels.

\section{REFERENCES}

[1] K. Abboud, H. A. Omar, and W. Zhuang, "Interworking of DSRC and Cellular Network Technologies for V2X Communications: A Survey," IEEE Transactions on Vehicular Technology, vol. 65 , no. 12 , pp. 9457-9470, 2016.

[2] R. F. Atallah, M. J. Khabbaz, and C. M. Assi, "Vehicular networking: A survey on spectrum access technologies and persisting challenges," Vehicular Communications, vol. 2, no. 3, pp. 125 - 149, 2015. [Online]. Available: http://www. sciencedirect.com/science/article/pii/S2214209615000339

[3] R. Molina-Masegosa and J. Gozalvez, "LTE-V for Sidelink 5G V2X Vehicular Communications: A New 5G Technology for Short-Range Vehicle-to-Everything Communications," IEEE Vehicular Technology Magazine, vol. 12, no. 4, pp. 30-39, 2017.

[4] O. Sadio, I. Ngom, and C. Lishou, "Controlling WiFi Direct Group Formation for Non-Critical Applications in C-V2X Network," IEEE Access, vol. 8, pp. 79 947-79 957, 2020.

[5] V. Mannoni, V. Berg, S. Sesia, and E. Perraud, "A Comparison of the V2X Communication Systems: ITS-G5 and C-V2X," in 2019 IEEE 89th Vehicular Technology Conference (VTC2019Spring), 2019, pp. 1-5.

[6] K. Z. Ghafoor, M. Guizani, L. Kong, H. S. Maghdid, and K. F. Jasim, "Enabling Efficient Coexistence of DSRC and C-V2X in Vehicular Networks," IEEE Wireless Communications, vol. 27, no. 2, pp. 134-140, 2020.

[7] P. Wang, B. Di, H. Zhang, K. Bian, and L. Song, "Cellular V2X Communications in Unlicensed Spectrum: Harmonious Coexistence With VANET in 5G Systems," IEEE Transactions on Wireless Communications, vol. 17, no. 8, pp. 5212-5224, 2018.

[8] "Intelligent Transport Systems (ITS); Radiocommunications equipment operating in the $5855 \mathrm{MHz}$ to $5925 \mathrm{MHz}$ frequency band; Harmonised Standard covering the essential requirements of article 3.2 of Directive 2014/53/EU," ETSI EN 302 571 V2.1.1, Feb 2017. [Online]. Available: https://www.etsi.org/deliver/etsi_en/302500_302599/ 302571/02.01.01_60/en_302571v020101p.pdf

[9] 5GAA, "Coexistence of C-V2X and ITS-G5 at 5.9GHz," 2017. [Online]. Available: https://5gaa.org/wp-content/uploads/ 2017/08/5GAA_Position_Paper.pdf

[10] M. Nikowitz, Fully Autonomous Vehicles: Visions of the Future Or Still Reality? : Benefits, Challenges, Technical Requirements, Practical Examples, Future Outlook. epubli $\mathrm{GmbH}$, 2015. [Online]. Available: https://books.google.fi/ books?id=C0TejgEACAAJ

[11] B. Fleming, "New Automotive Electronics Technologies [Automotive Electronics]," IEEE Vehicular Technology Magazine, vol. 7, no. 4, pp. 4-12, 2012.

[12] D. Shamoto, A. Tomoeda, R. Nishi, and K. Nishinari, "Car-following model with relative-velocity effect and its experimental verification," Phys. Rev. E, vol. 83, p. 046105, Apr 2011. [Online]. Available: https://link.aps.org/doi/10.1103/ PhysRevE.83.046105

[13] Y. J. Cho, K. Huang, and C. Chae, "V2X Downlink Coverage Analysis with a Realistic Urban Vehicular Model," in 2018 IEEE Globecom Workshops (GC Wkshps), 2018, pp. 1-6.

[14] Y. S. Song, S. K. Lee, J. W. Lee, D. W. Kang, and K. W. Min, "Analysis of adjacent channel interference using distribution function for V2X communication systems in the $5.9-\mathrm{GHz}$ band for ITS," ETRI Journal, vol. 41, no. 6, pp. 703-714, 2019.

[15] A. Bazzi, G. Cecchini, M. Menarini, B. M. Masini, and A. Zanella, "Survey and perspectives of vehicular wi-fi versus sidelink cellular-v2x in the $5 \mathrm{~g}$ era," Future Internet, vol. 11, no. 6 , p. $122,2019$.

[16] Technical Specification Group Radio Access Network, "Study on LTE-Based V2X Services," 3GPP TR 36.885 V14.0.0, 2016. [Online]. Available: https://www.3gpp.org/dynareport/36885. htm 